\title{
On the Energy Efficiency of Hybrid Relaying Schemes in the Two-way Relay Channel
}

\author{
Yinan Qi, Muhamamd Ali Imran, and Rahim Tafazolli \\ Centre for Communication Systems Research, University of Surrey, Guildford, United Kingdom
}

\begin{abstract}
In this paper, hybrid relaying schemes are investigated in the two-way relay channel, where the relay node is able to adaptively switch between different forwarding schemes based on the current channel state and its decoding status and thus provides more flexibility as well as improved performance. The analysis is conducted from the energy efficiency perspective for two transmission protocols distinguished by whether exploiting the direct link between two main communicating nodes (the source and destination nodes, and vice versa since it is two way communication) or not. A realistic power model taking circuitry power consumption of all involved nodes into account is employed. The energy efficiency is optimized in terms of consumed energy per bit subject to the Quality of Service (QoS) constraint. Numerical results show that the hybrid schemes are able to achieve the highest energy efficiency due to its capability of adapting to the channel variations and the protocol where the direct link is exploited is more energy efficient.
\end{abstract}

Keywords: Energy efficiency, two-way relay channel, network coding, compress-and-forward, outage probability

\section{Introduction}

Global warming has become a more and more serious challenge across the whole world in the past century mostly caused by human activities that increase concentrations of greenhouse gases in the atmosphere. Some scientific findings reveal that the $\mathrm{CO}_{2}$ emission of the ICT (Information \& Communications Technology) industry has contributed to a considerable percentage to the world energy consumption budget and global warming $[1,2]$. The arising challenge of the huge increase of energy consumption in the face of the exploding traffic demands of future communication systems motivates the need for greener and more energy efficient techniques. Among all the candidates, relaying technique has become increasingly important, motivated by its capability to provide uniform high coverage and potential energy savings.

The relay model is firstly introduced by van der Meulen [3] and substantially developed by Cover and El Gamal in their landmark work [4]. While the optimal relaying strategy in wireless networks has not yet been fully understood, several relaying schemes have been suggested in the literature including amplify-and-forward (AF), decode-andforward (DF) and compress-and-forward (CF) [5, 6]. More recently, advanced hybrid relaying schemes have been proposed and analyzed from both spectrum and energy efficiency perspectives [7]. However, these works are focused on one way relay where the communication is in one direction only, i.e. from the source to the destination. The communication systems, in nature, are two-way systems where two terminals transmit to each other simultaneously, i.e. a source can also be a destination. In this regard, a twoway relay channel (TWRC), where two nodes communicate with each other via a relay node, has drawn more and more attention recently. The well developed relaying strategies have been applied in the TWRC in $[5,6]$ for both fullduplex and half-duplex systems. A novel coding scheme called network coding (NC), where instead of simply relaying the received packets of information, the relay node take two packets from two main nodes and combine then together for transmission, has been proposed and investigated in $[8,9]$.

The existing analysis on the TWRC is mainly aiming at spectrum efficiency analysis, such as achievable rate region [10] and capacity bounds [8]. On the contrary, this work exploits the energy efficiency perspective with a realistic power consumption model to optimize the energy usage of the entire system. In addition to transmission power, circuitry power consumption of all involved nodes is taken into consideration to give insight of how to design a practical energy efficient system. Moreover, in order to further exploit the degrees of freedom of a time-variant wireless media, conventional forwarding mechanisms and network coding schemes are combined in a hybrid fashion to better adapt to the channel variations. Specifically, CF is used in conjunction with $\mathrm{NC}$ in this work. We focus on more feasible half-duplex mode where simultaneously transmission and reception are forbidden at each node. Two transmission protocols are studied. The direct link between two main nodes is exploited in the first protocol and the entire frame is divided into three phases. On the other hand, the second protocol does not use the direct link and therefore each frame only consists of two phases (the detailed frame structure will be described later in section II).

The rest of the paper is organized as follows: in the next section, the system model is introduced and problem formulation is presented. Section III presents the derivation of the outage probability for different relaying strategies. The energy consumption model and energy efficiency analysis is studied in section IV where an energy efficiency objective function is also established. Numerical results are shown in section $\mathrm{V}$ and the final section concludes.

\section{System Model}

As shown in figure 1, two main nodes 1 and 2, denoted as $S_{1}$ and $S_{2}$, respectively, exchange information through the relay node $R$. When the direct link $S_{1}-S_{2}$ is exploited, the entire transmission can be separated into three phases as shown in figure 1(a): during the first phase, $S_{1}$ broadcasts and $S_{2}$ and $R$ receive. Since it is half-duplex mode, $S_{2}$ cannot transmit simultaneously in phase $1 . S_{2}$ broadcasts and $S_{1}$ and $R$ receive in the second phase. In the final phase, $R$ broadcasts to $S_{1}$ and $S_{2}$. Each phase lasts for $T / 3$ seconds, where $T$ is the activation time and subject to the constraint 


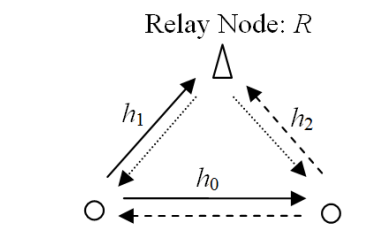

Main Node: $S_{1} \quad$ Main Node: $S_{2}$ (a)

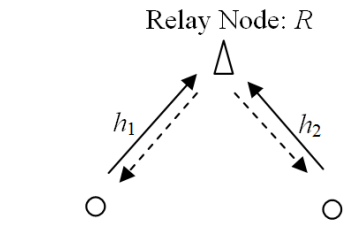

Main Node: $S_{1} \quad$ Main Node: $S_{2}$ (b)

Phase 3

Fig.1 Two-way relay channel: $(a)$ three-phase case $(b)$ two-phase case. $T \leq T_{\max }$. The signals received at $S_{2}$ and $R$ during phase 1 are given by

$$
y_{2}^{\langle 1\rangle}(t)=h_{0} x_{1}(t)+n_{2}^{\langle 1\rangle}(t), y_{r}^{\langle 1\rangle}(t)=h_{1} x_{1}(t)+n_{r}^{\langle 1\rangle}(t),
$$

with $h_{i}=\frac{c_{i}}{\eta d_{i}^{\xi / 2}}$, for $i=01,2$, where the superscripts in angle brackets indicate phases (only used when necessary), $x_{1}$ is the transmitted signal from $S_{1}, n_{2}$ and $n_{r}$ are the noise at $S_{1}$ and $R$, respectively, following circularly symmetric complex Gaussian (CSCG) distribution with zero mean, $c_{i}$ represents fast fading of link $i$ and follows CSCG distribution with unit variance and zero mean, $d_{i}$ is the distance of link $i, \xi$ is the path loss exponent, and $\eta$ is a constant indicating the physical characteristics of the link and the power amplifier as in [7]. Similarly, the signal received during phase 2 are given by

$$
y_{1}^{\langle 2\rangle}(t)=h_{0} x_{2}(t)+n_{1}{ }^{\langle 2\rangle}(t), y_{r}^{\langle 2\rangle}(t)=h_{2} x_{2}(t)+n_{r}{ }^{\langle 2\rangle}(t),
$$

at $S_{2}$ and $R$, respectively, where $x_{2}$ is the $S_{2}$ signal, and $n_{1}$ is the noise at $S_{1}$, following the same distribution as $n_{r}$. The received two signals from two main nodes are processed at the relay node and a new signal is generated and broadcasted to $S_{1}$ and $S_{2}$ by $R$ in the final phase. The received signals are

$$
y_{1}^{\langle 3\rangle}(t)=h_{1} x_{r}(t)+n_{1}{ }^{\langle 3\rangle}(t), y_{2}^{\langle 3\rangle}(t)=h_{2} x_{r}(t)+n_{2}{ }^{\langle 3\rangle}(t),
$$

respectively.

On the contrary, when the direct link $S_{1}-S_{2}$ is neglected, the entire transmission can be separated into two phases: $R$ receiving phase and $R$ broadcasting phase as shown in figure $1(b)$. Each phase lasts for $T / 2$ seconds. The signal received at $R$ during phase 1 is given by

$$
y_{r}(t)=h_{1} x_{1}(t)+h_{2} x_{2}(t)+n_{r}(t),
$$

During phase $2, R$ broadcasts and the received signal at $S_{1}$ and $S_{2}$ are

$$
y_{1}(t)=h_{1} x_{r}(t)+n_{1}(t), y_{2}(t)=h_{2} x_{r}(t)+n_{2}(t),
$$

respectively.

The objective of the optimization is to minimize the energy expenditure for each transmitted information bit. However, this optimization should be subject to some Quality of Service (QoS) constraints to guarantee that the user experience is maintained at a satisfactory level. As we know, outage probability indicates the possibility of unsuccessful reception and can be used as a QoS constraint in this work. We define the consumed transmission energy for each bit as $E_{b}$. Clearly, outage probability should a function of $E_{b}$ and the activation time $T$, and thus can be expressed as $p\left(E_{b}, T\right)$. Considering there are three nodes in the TWRC, each nodes might have different level of energy expenditure and the outage probability should be rewritten as $p\left(E_{b, 1}, E_{b, 2}, P_{r}, T\right)$, where $P_{r}$ is the transmission power of node $R$. Let's define the overall energy consumption of the entire system including circuitry power expenditure for each bit as $E$. It is straightforward to see that $E$ should be a function of $E_{b, 1}, E_{b, 2}$ and $P_{r}$ as well. It is also a function of activation time $T$ because circuitry energy consumption is depending on it. Thus it takes the form as $E\left(E_{b, 1}, E_{b, 2}, P_{r}, T\right)$. Now the problem is to minimize the overall average energy consumption per bit $E$ given the target outage probability $p_{t}$ and maximum activation time $T_{\max }$. It can be mathematically formulated as

$$
\begin{array}{ll}
\text { minimize } & E\left(E_{b, 1}, E_{b, 2}, P_{r}, T\right) \\
\text { subject to } & p\left(E_{b, 1}, E_{b, 2}, P_{r}, T\right) \leq p_{t} . \\
& T \leq T_{\max }
\end{array}
$$

The main contribution presented in this work is to solve this energy efficiency optimization problem in the TWRC for hybrid relaying schemes.

\section{Outage Analysis}

As we mentioned, outage probability $p\left(E_{b, 1}, E_{b, 2}, P_{r}, T\right)$ will be used as the QoS constraint when minimizing the energy consumption. In this section, we will investigate the outage performance of different relaying schemes.

We assume that the message from node $S_{1}$ to $S_{2}$ is $m_{1}$ belonging to a set $\Omega_{1}=\left\{1, \ldots, 2^{L_{1}}\right\}$, and message $m_{2}$ in the reverse direction belonging to a set $\Omega_{2}=\left\{1, \ldots, 2^{L_{2}}\right\}$. The transmission power of node $S_{1}$ and $S_{2}$ are $P_{1}$ and $P_{2}$, respectively. Here for simplicity, we assume that $P_{1}=P_{2}=P_{s}$. In this regard, we have $L_{1} E_{b, 1} / T=L_{2} E_{b, 2} / T$. In such a case, $E_{b, 1}$ can be eliminated from the overall energy consumption $E$ and outage probability functions.

Network Coding (NC): In three-phase NC, the relay node firstly tries to decode two messages received in two separate phases before re-encoding them with network coding technique. During the first phase, the message broadcasted by $S_{1}$ can be decoded successfully at node $R$ if

$$
R_{N C, 1 \rightarrow R}^{3}=\frac{1}{3} C\left(\frac{3 \gamma_{1} E_{b, 1} L_{1}}{N_{0} B T}\right) \geq \frac{L_{1}}{T},
$$

where $C(x)=B \log _{2}(1+x)$ and the superscript of $R_{N C, 1 \rightarrow R}^{3}$ indicates that it is three-phase operation, $\gamma_{i}=\left|h_{i}\right|^{2}, N_{0}$ is the single-sided thermal noise spectral density and $B$ (in Hertz) stands for the system bandwidth. Similarly, message $m_{2}$ can be decoded as long as

$$
R_{N C, 2 \rightarrow R}^{3}=\frac{1}{3} C\left(\frac{3 \gamma_{2} E_{b, 2} L_{2}}{N_{0} B T}\right) \geq \frac{L_{2}}{T} .
$$

Multiplexed coding [8] is then used for $\mathrm{NC}$ at node $R$. Without loss of generality, we assume that $L_{1} \leq L_{2}$. We first set up a mapping from $\Omega_{2}$ to $2^{L_{2}} L_{2}$-dimentional vectors in the finite field of size $2 \mathrm{~F}_{2}{ }^{L_{2}}$. Then we associate each vector with an independent Gaussian codeword. We do the same mapping and codeword generation and association for $\Omega_{1}$. The only difference is that the mapping is to a sub-space of $\mathrm{F}_{2}{ }^{{ }_{2}}$, denoted as $\mathrm{F}_{\text {sub }}$. The relay node transmits the codeword associated with $m_{1}+m_{2}$, where the addition is performed using arithmetics in field $\mathrm{F}_{2}{ }^{{ }_{2}}$. During phase 3, $R$ broadcasts a network coded Gaussian codeword $x_{r}\left(m_{1}+m_{2}\right)$ to $S_{1}$ and $S_{2}$. Upon reception of $x_{r}\left(m_{1}+m_{2}\right), S_{1}$ tries to decode $m_{1}+m_{2}$ by jointly processing $y_{1}^{\langle 3\rangle}$ and its reception $y_{1}^{\langle 2\rangle}$ during the second phase. It can do so if

$$
R_{N C, 2 \rightarrow 1}^{3}=\frac{1}{3} C\left(\frac{3 \gamma_{0} E_{b, 2} L_{2}}{N_{0} B T}\right)+\frac{1}{3} C\left(\frac{\gamma_{1} P_{r}}{N_{0} B}\right) \geq \frac{L_{2}}{T} .
$$

Then it subtracts the vector corresponding to $m_{1}$ from the decoded message to get $m_{2}$. In node $S_{2}, m_{2}$ is known as well as the associated affine space $m_{2}+\mathrm{F}_{s u b}$. It only needs to find the codeword in this affine space. By taking $y_{2}^{\langle 1\rangle}$ into account, it can successfully decode when

$$
R_{N C, 1 \rightarrow 2}^{3}=\frac{1}{3} C\left(\frac{3 \gamma_{0} E_{b, 1} L_{1}}{N_{0} B T}\right)+\frac{1}{3} C\left(\frac{\gamma_{2} P_{r}}{N_{0} B}\right) \geq \frac{L_{1}}{T} .
$$


Then $m_{2}$ is subtracted to get $m_{1}$. Since there are two messages exchanged between $S_{1}$ and $S_{2}$, unsuccessful receptions can happen at either node. In this work, if either $S_{1}$ or $S_{2}$ detects errors, outage is claimed. In order to derive the outage probability, we can first look at the successful decoding probability which is complementary to outage probability. As we can see, correct reception requires both the relay node and two main nodes to successfully decode the message and outage probability can be expressed as

$$
p\left(E_{b, 1}, P_{r}, T\right)=1-\operatorname{Pr}\{\text { successful decoding }\}
$$

$$
=1-\operatorname{Pr}\left\{\begin{array}{l}
R_{N C, 1 \rightarrow R}^{3} \geq \frac{L_{1}}{T}, R_{N C, 2 \rightarrow R}^{3} \geq \frac{L_{2}}{T}, \\
R_{N C, 2 \rightarrow 1}^{3} \geq \frac{L_{2}}{T}, R_{N C, 1 \rightarrow 2}^{3} \geq \frac{L_{1}}{T}
\end{array}\right\}
$$

The outage probability of two-phase NC can be derived following the same line of argument and the derivation is given in Appendix A.

Hybrid $N C / C F$ : In NC, the relay node needs to be able to decode two received messages before employing multiplexed coding. However, due to the time variation characteristics of the wireless media successful decoding might not be achieved when deep fading occurs. Here we introduce more flexibility to the relay's forwarding scheme by allowing CF operation when decoding fails at node $R$. For the three-phase scenario, we need to consider four kinds of decoding status as follows:

a) Message $m_{1}$ is decoded but $m_{2}$ is not. The relay node re-encodes $m_{1}$ and quantizes its phase 2 reception $y_{r}^{\langle 2\rangle}$ into intermediate bin index $u$, which is then compressed to index $v$ [11].The minimum number of compressed information bits for $v$, such that the compressed signal can be recovered with the smallest distortion, is denoted as $s$ and is given by

$$
\begin{aligned}
s & =\frac{1}{3} T B\left(H\left(W \mid y_{1}^{\langle 2\rangle}\right)-H\left(W \mid y_{r}^{\langle 2\rangle}\right)\right) \\
& =\frac{1}{3} T B \log _{2}\left(\frac{\gamma_{2} P_{s}+N_{0} B+\sigma_{W}^{2}-\frac{\gamma_{0} \gamma_{2} P_{s}^{2}}{\gamma_{0} P_{s}+N_{0} B}}{\sigma_{W}^{2}}\right),
\end{aligned}
$$

where $W$ is an auxiliary random variable with variance $\sigma_{W}^{2}$. Finally, this index $v$ is encoded as $x_{c}(v)$ and added together with the re-encoded message $x_{1}\left(m_{1}\right)$ to form the relay node's transmitted signal as

$$
x_{r}=\beta_{a} x_{\mathrm{c}}+\beta_{b} x_{1} .
$$

with power constraint $\beta_{a}^{2} P_{c}+\beta_{b}^{2} P_{s} \leq P_{r}$. At node $S_{1}$, the known part $\beta_{b} h_{1} x_{1}$ is cancelled from its reception $y_{1}^{\langle 3\rangle}$ and $v$ can be decoded if

$$
R_{H i, R \rightarrow 1}^{3}=\frac{1}{3} C\left(\frac{\beta_{a}^{2} \gamma_{1} P_{c}}{N_{0} B}\right) \geq \frac{s}{T} .
$$

Once $v$ is obtained, with the help of side information $y_{1}^{\langle 2\rangle}$, the estimation of the relay node's reception can be reconstructed and denoted as $\hat{y}_{r}^{\langle 2\rangle}$. With joint processing of $y_{1}^{\langle 2\rangle}$ and $\hat{y}_{r}^{\langle 2\rangle}$, node $S_{1}$ can decode message $m_{2}$ as long as [6]

$$
R_{H i, 2 \rightarrow 1}^{3}=\frac{1}{3} C\left(\frac{3 \gamma_{0} E_{b, 2} L_{2}}{N_{0} B T}+\frac{3 \gamma_{2} E_{b, 2} L_{2}}{\left(N_{0} B+\sigma_{W}^{2}\right) T}\right) \geq \frac{L_{2}}{T} \text {. }
$$

where $\sigma_{W}^{2}$ can be obtained from (3) and (4). At node $S_{2}$, $\beta_{a} h_{2} x_{c}$ is treated as noise. Based on joint processing of $y_{2}^{\langle 1\rangle}$ and $y_{2}^{\langle 3\rangle}, m_{1}$ can be successfully decoded if

$$
R_{H i, 1 \rightarrow 2}^{3}=\frac{1}{3} C\left(\frac{3 \gamma_{0} E_{b, 1} L_{1}}{N_{0} B T}\right)+\frac{1}{3} C\left(\frac{3 \gamma_{2} \beta_{b}^{2} E_{b, 1} L_{1}}{\left(\beta_{a}^{2} \gamma_{2} P_{c}+N_{0} B\right) T}\right) \geq \frac{L_{1}}{T} .
$$

The successful decoding probability of both messages is given as

$$
p_{a}\left(E_{b, 1}, P_{r}, T\right)=\operatorname{Pr}\left\{\begin{array}{l}
R_{N C, 1 \rightarrow R}^{3} \geq \frac{L_{1}}{T}, R_{N C, 2 \rightarrow R}^{3}<\frac{L_{2}}{T}, \\
R_{H i, 2 \rightarrow 1}^{3} \geq \frac{L_{2}}{T}, R_{H i, 1 \rightarrow 2}^{3} \geq \frac{L_{1}}{T}
\end{array}\right\} .
$$

b) Message $m_{2}$ is decoded but $m_{1}$ is not. The decoding probability $p_{b}$ can be derived following the same line of argument.

c) Neither message $m_{1}$ nor $m_{2}$ is decoded. The relay node simply compresses the summation of $y_{r}^{\langle 1\rangle}$ and $y_{r}^{\langle 2\rangle}$. Similar compression/decompression procedure as described in case $(a)$ is employed at both main nodes. Message $m_{1}$ and $m_{2}$ can be decoded if

$$
\begin{aligned}
& R_{C F, 2 \rightarrow 1}^{3}=\frac{1}{3} C\left(\frac{3 \gamma_{0} E_{b, 2} L_{2}}{N_{0} B T}+\frac{3 \gamma_{2} E_{b, 2} L_{2}}{\left(2 N_{0} B+\sigma_{W}^{2}\right) T}\right) \geq \frac{L_{2}}{T}, \\
& R_{C F, 1 \rightarrow 2}^{3}=\frac{1}{3} C\left(\frac{3 \gamma_{0} E_{b, 1} L_{1}}{N_{0} B T}+\frac{3 \gamma_{1} E_{b, 1} L_{1}}{\left(2 N_{0} B+\sigma_{W}^{2}\right) T}\right) \geq \frac{L_{1}}{T} .
\end{aligned}
$$

where the compression noise can be obtained by the constraint [10]

$$
\begin{array}{r}
\max \left\{H\left(W \mid y_{2}^{\langle 1\rangle}\right)-H\left(W \mid y_{r}^{\langle 1\rangle}\right), H\left(W \mid y_{1}^{\langle 2\rangle}\right)-H\left(W \mid y_{r}^{\langle 2\rangle}\right)\right\} \\
\leq \frac{1}{T B} \min \left\{C\left(\frac{\gamma_{1} P_{r}}{N_{0} B}\right), C\left(\frac{\gamma_{2} P_{r}}{N_{0} B}\right)\right\}
\end{array}
$$

It follows that the decoding probability is given as

$$
p_{c}\left(E_{b, 1}, P_{r}, T\right)=\operatorname{Pr}\left\{\begin{array}{l}
R_{N C, 1 \rightarrow R}^{3}<\frac{L_{1}}{T}, R_{N C, 2 \rightarrow R}^{3}<\frac{L_{2}}{T}, \\
R_{C F, 2 \rightarrow 1}^{3} \geq \frac{L_{2}}{T}, R_{C F, 1 \rightarrow 2}^{3} \geq \frac{L_{1}}{T}
\end{array}\right\}
$$

d) Both messages are decoded. The decoding probability $p_{d}$ is given as

$$
p_{d}\left(E_{b, 1}, P_{r}, T\right)=\operatorname{Pr}\left\{\begin{array}{l}
R_{N C, 1 \rightarrow R}^{3} \geq \frac{L_{1}}{T}, R_{N C, 2 \rightarrow R}^{3} \geq \frac{L_{2}}{T}, \\
R_{N C, 2 \rightarrow 1}^{3} \geq \frac{L_{2}}{T}, R_{N C, 1 \rightarrow 2}^{3} \geq \frac{L_{1}}{T}
\end{array}\right\} .
$$

Since four events are mutually exclusive, it follows that the overall outage probability is $p\left(E_{b, 1}, P_{r}, T\right)=1-\left(p_{a}+p_{b}+p_{c}+p_{d}\right)$. The outage probability of two-phase case is derived in Appendix B.

\section{Energy Efficiency Analysis}

The objective of the energy efficiency optimization is to minimize the overall energy expenditure including circuitry power consumption per transmitted information bit. In the three-phase case, during the first phase node $S_{1}$ broadcasts and nodes $S_{2}$ and $R$ receive. The consumed energy for all three nodes is given as

$$
E^{\langle 1\rangle}=L_{1} E_{b, 1}+\left(P_{c t}+2 P_{c r}\right) T / 3,
$$

where $P_{c t}$ and $P_{c r}$ are the transmission and reception circuitry power, respectively. The first term represents 


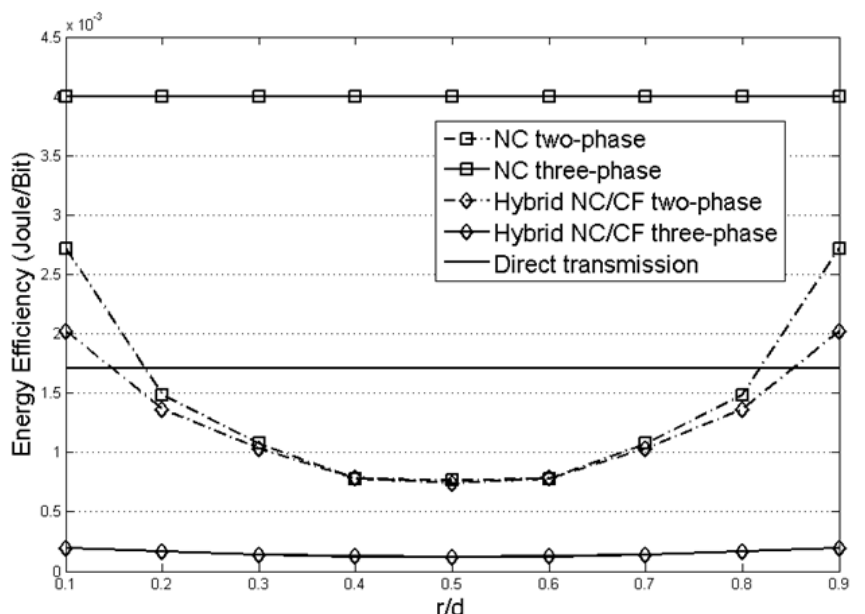

Fig. 2 Energy Efficiency, $d=100 \mathrm{~m}$.

transmission power of $S_{1}$ and the second term stands for the circuitry power consumption of one transmitting node $\left(S_{1}\right)$ and two receiving node $\left(S_{2}\right.$ and $R$ ). During phase 2, $S_{2}$ broadcasts to $S_{1}$ and $R$ and the associated energy consumption is expressed as

$$
E^{\langle 2\rangle}=L_{2} E_{b, 2}+\left(P_{c t}+2 P_{c r}\right) T / 3,
$$

where the first term is the $S_{2}$ transmission power and the last term is circuitry power consumption. In the final phase, node $R$ broadcasts and power consumption is given by

$$
E^{\langle 3\rangle}=P_{r} T / 3+\left(P_{c t}+2 P_{c r}\right) T / 3,
$$

where the first term is the relay node transmission power consumption. The overall energy consumed for transmitting message $m_{1}$ and $m_{2}$ is

$$
\mathrm{E}\left(E_{b, 1}, E_{b, 2}, T, P_{r}\right)=E^{\langle 1\rangle}+E^{\langle 2\rangle}+E^{\langle 3\rangle} .
$$

In order to optimize the energy efficiency, we set the objective function as

$$
\begin{array}{ll}
\text { minimize } & E\left(E_{b, 1}, T, P_{r}\right)=\frac{\mathrm{E}\left(E_{b, 1}, T, P_{r}\right)}{L_{1}+L_{2}} \\
\text { subject to: } & p\left(E_{b, 1}, P_{r}, T\right) \leq p_{t} \\
& T \leq T_{\max }
\end{array} .
$$

From the outage probability expressions, it is easy to see that with fixed $L_{1}$ and $L_{2}$, the outage probability is a function of $E_{b, 1}$, relay transmission power $P_{r}$, and activation time $T$. Once we fix the outage probability at target one, $E_{b, 1}$ can be expressed as a function of $P_{r}$ and $T$, which makes $E\left(E_{b, 1}\left(P_{r}\right.\right.$, $\left.T), P_{r}, T\right)$ a function of $P_{r}$ and $T$ as well. It means that the minimum energy per bit can be optimized by manipulating $P_{r}$ and $T$. This problem can be solved numerically.

In the two-phase case, the energy consumption for each phase can be easily derived and given as

$$
\begin{aligned}
& E^{\langle 1\rangle}=L_{1} E_{b, 1}+L_{2} E_{b, 2}+\left(2 P_{c t}+P_{c r}\right) T / 2, \\
& E^{\langle 2\rangle}=P_{r} T / 2+\left(2 P_{c r}+P_{c t}\right) T / 2,
\end{aligned}
$$

respectively. The optimization problem can be formulated in the same manner as in (8).

\section{Numerical Results and Discussions}

In this section, we compare the energy efficiency performance of different schemes. We use the following settings: $L_{1}=L_{2}=10, P_{c t}=98 \mathrm{~mW}, P_{c r}=112.4 \mathrm{~mW}, \eta=7.78 \times 10^{4}$, $\zeta=3, \quad N_{0}=-171 \mathrm{dBm} / \mathrm{Hz}, \quad B=1, \quad \rho=0.5$ and $T_{\max }=10$. The distance between $S_{1}$ and $S_{2}$ is $d$ and the distance between $S_{1}$ and $R$ is expressed by $r$. Three nodes are assumed to be in a straight line with $R$ in the middle and moving towards $S_{2}$ from $S_{1}$.

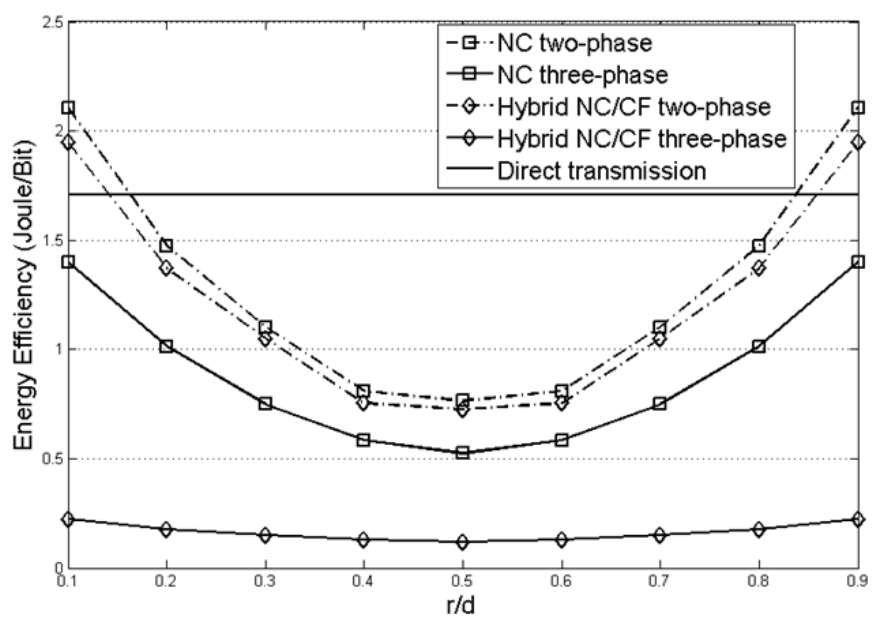

Fig. 3 Energy Efficiency, $d=1000 \mathrm{~m}$.

Figure 2 shows the energy efficiency performance in terms of consumed energy per bit in short transmission range $(d=100 \mathrm{~m})$. We see that hybrid schemes demonstrate better energy efficiency performance compared to $\mathrm{NC}$ and direct transmission due to its flexibility (except the extremely asymmetric case in the two-phase scenario, where $R$ is very close to either $S_{1}$ or $S_{2}$ ). For three-phase schemes, their consumed energy per bit is almost irrelevant with the position of node $R$. This is caused by the fact that two nodes mainly communicate through the direct link since their distance is small and the link quality is naturally high. Using a relaying node usually helps to improve the outage behaviour. With a fixed outage target, it means lower transmission power consumption. However, when circuitry power consumption is taken into account, especially in short distance, where it accounts for a major part of overall energy expenditure, the additional energy consumption of the relay node might overweight the benefit of improved outage behaviour. This is why direct transmission outperforms twophase NC in this relatively short distance. Another interesting observation is that the best energy efficiency is achieved by the three-phase hybrid scheme. When the transmission range is increased to $1000 \mathrm{~m}$, as shown in figure 3 , most of the relaying strategies totally or partly outperform direct transmission because the transmission energy is the main part of the total energy consumption and adding a relay node can significantly reduce the transmission power. Hybrid schemes still out perform NC and achieve much high energy efficiency compared to direct transmission. The best energy efficiency is achieved by the three-phase hybrid one.

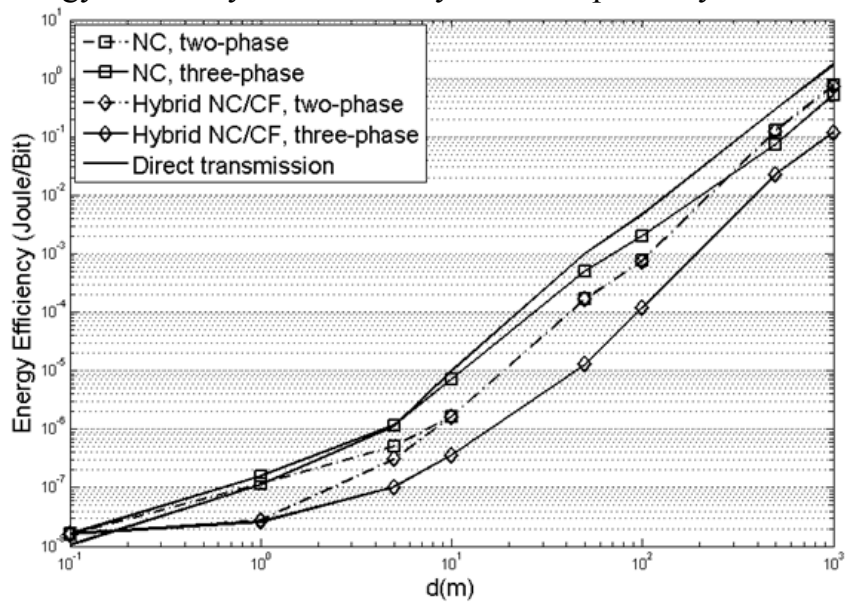

Fig. 4 Energy efficiency when $d$ is increasing.

Figure 4 shows the energy efficiency when the distance between two main nodes increases from 0.1 to $1000 \mathrm{~m}$. Node $R$ is in the middle of two nodes. Direct transmission achieves the best performance in very short distance; while 
three-phase hybrid $\mathrm{NC} / \mathrm{CF}$ scheme has the lowest energy expenditure when the distance is increased.

\section{Conclusions and Future Work}

In this paper, we investigate, with a practical power model, the energy efficiency performance of hybrid relaying strategies switching between $\mathrm{NC}$ and $\mathrm{CF}$ in the two-way relay channel. Two system settings are assumed: with or without exploiting the direct link and the frame structure is divided into two and three phases, respectively. The energy efficiency in terms of energy per bit is minimized by manipulating the transmission power and the activation time of all involved nodes. The results show that the hybrid schemes which enjoy more flexibility and are able to adapt to the channel variation outperform non-hybrid ones. However, this improvement is only achieved when the distance between two main nodes is not very close and the circuitry power consumption accounts for only a small part of the overall energy expenditure. An interesting observation is that, compared to the two-phase hybrid relaying scheme, the three-phase hybrid one achieves a better energy efficiency performance by exploiting the direct link between two main nodes.

In the current settings, we only consider balanced scenario where the transmission powers of $S_{1}$ and $S_{2}$ are the same, non-balanced cases will be addressed in the future. In addition, the application of multiple relay nodes is also an interesting topic to study in our future work.

\section{Appendix A}

For two-phase NC, the first phase is a MAC channel and two messages can be decode successfully at node $R$ if [12]

$$
\begin{aligned}
& R_{N C, 1 \rightarrow R}^{2}=\frac{1}{2} C\left(\frac{2 \gamma_{1} E_{b, 1} L_{1}}{N_{0} B T}\right) \geq \frac{L_{1}}{T}, R_{N C, 2 \rightarrow R}^{2}=\frac{1}{2} C\left(\frac{2 \gamma_{2} E_{b, 2} L_{2}}{N_{0} B T}\right) \geq \frac{L_{2}}{T}, \\
& R_{N C, 12 \rightarrow R}^{2}=\frac{1}{2} C\left(\frac{2 \gamma_{1} E_{b, 1} L_{1}+2 \gamma_{2} E_{b, 2} L_{2}}{N_{0} B T}\right) \geq \frac{L_{1}+L_{1}}{T} .
\end{aligned}
$$

Multiplexed coding can also be used here and the same decoding procedure is employed in both main nodes. The messages can be decoded if

$$
R_{N C, R \rightarrow 1}^{2}=\frac{1}{2} C\left(\frac{2 \gamma_{1} P_{r}}{N_{0} B}\right) \geq \frac{L_{2}}{T}, R_{N C, R \rightarrow 2}^{2}=\frac{1}{2} C\left(\frac{2 \gamma_{2} P_{r}}{N_{0} B}\right) \geq \frac{L_{1}}{T} .
$$

The outage probability can be given in a similar formation as in (2).

\section{Appendix B}

In two-phase hybrid scheme, the fact that only message $m_{1}$ is decoded during the first phase means [12]

$$
R_{N C, 2 \rightarrow R}^{2}<\frac{L_{2}}{T}, \frac{1}{2} C\left(\frac{2 \gamma_{1} E_{b, 1} L_{1}}{\left(N_{0} B+P_{s}\right) T}\right) \geq \frac{L_{1}}{T} .
$$

The relay subtracts $h_{1} x_{1}$ from the received signal, reencodes $m_{1}$ and compresses the residual signal $\bar{y}_{r}(t)=h_{2} x_{2}(t)+n_{r}(t)$. Then it broadcasts the weighted summation as $x_{r}=\beta_{a} x_{c}+\beta_{b} x_{1}$. At node $S_{1}$, the known part $\beta_{b} h_{1} x_{1}$ is cancelled and tries to reconstruct estimation of $\bar{y}_{r}$. If can do so if [13]

$$
I\left(\bar{y}_{r} ; \hat{\bar{y}}_{r}\right) \leq \frac{1}{2 B T} C\left(\frac{\beta_{a}^{2} \gamma_{1} P_{c}}{N_{0} B}\right) .
$$

It follows that $m_{2}$ can be decoded if

$$
R_{H i, 2 \rightarrow 1}^{2}=\frac{1}{2} C\left(\frac{2 \gamma_{2} E_{b, 2} L_{2}}{\left(N_{0} B+\sigma_{W}^{2}\right) T}\right) \geq \frac{L_{2}}{T} .
$$

At node $S_{2}, \beta_{a} h_{2} x_{c}$ is treated as noise. The message $m_{1}$ can be decoded if

$$
R_{H i, 1 \rightarrow 2}^{2}=\frac{1}{2} C\left(\frac{2 \gamma_{2} \beta_{b}^{2} E_{b, 1} L_{1}}{\left(N_{0} B+\beta_{a}^{2} \gamma_{2} P_{c}\right) T}\right) \geq \frac{L_{1}}{T} .
$$

The successful decoding probability is given as

$$
p_{a}\left(E_{b, 1}, P_{r}, T\right)=\operatorname{Pr}\left\{\begin{array}{l}
R_{N C, 2 \rightarrow R}^{2}<\frac{L_{2}}{T}, \frac{1}{2} C\left(\frac{2 \gamma_{1} E_{b, 1} L_{1}}{\left(N_{0} B+P_{s}\right) T}\right) \geq \frac{L_{1}}{T}, \\
R_{H i, 2 \rightarrow 1}^{2} \geq \frac{L_{2}}{T}, R_{H i, 1 \rightarrow 2}^{2} \geq \frac{L_{1}}{T}
\end{array}\right\} .
$$

Probability $p_{b}$ and $p_{c}$ can be derived in a similar way and $p_{d}$ is given by

$$
p_{d}\left(E_{b, 1}, P_{r}, T\right)=\operatorname{Pr}\left\{\begin{array}{l}
R_{N C, 1 \rightarrow R}^{2} \geq \frac{L_{1}}{T}, R_{N C, 2 \rightarrow R}^{2} \geq \frac{L_{2}}{T}, R_{N C, 12 \rightarrow R}^{2} \geq \frac{L_{1}+L_{1}}{T}, \\
R_{N C, 2 \rightarrow 1}^{2} \geq \frac{L_{2}}{T}, R_{N C, 1 \rightarrow 2}^{2} \geq \frac{L_{1}}{T}
\end{array}\right\} .
$$

The overall outage probability is $p\left(E_{b, 1}, P_{r}, T\right)=1$ $\left(p_{a}+p_{b}+p_{c}+p_{d}\right)$.

\section{Acknowledgement}

The research leading to these results has received funding from the European Community's Seventh Framework Programme [FP7/2007-2013] under grant agreement $\mathrm{n}^{\circ} 247733$ - project EARTH.

\section{References}

[1] https://www.ict-earth.eu/Earth project Summary leaflet.

[2] Press release, EU Commissioner Calls on ICT Industry to Reduce Its Carbon Footprint by 20\% as Early as 2015, MEMO/09/140, Mar. 2009.

[3] E.C. van der Meulen, "Three-terminal Communication Channel," Adv. Appl. Prob., vol. 3, pp. 120-154, 1971.

[4] Cover, T. and Gamal, A.E. "Capacity Theorems for the Relay Channel," IEEE Trans. Inform. Theory, vol. 25, pp. 572-584, Sep. 1979.

[3] C. E. Shannon, "Two-way Communication Channels," in Proc. of the $4^{\text {th }}$ Berkeley Symposium on Mathematical Statistics and Probability, pp. 611-644, Jun. 1961.

[4] B. Rankov and A. Wittneben, "Achievable Rate Region for the Two-way Relay Channel," in Proc. of ISIT'06, pp.1668-1672, Jul. 2006.

[5] M. Janani, A. Hedayat, T. E. Hunter, and A. Nosratinia, "Coded cooperation in wireless communications: Space-time transmission and iterative decoding," IEEE Trans. Signal Process., vol. 52, no. 2, pp. 362371, Feb. 2004.

[6] A. Host-Madsen and J. Zhang, "Capacity bounds and power allocation for wireless relay channels," IEEE Trans. Inform. Theory, vol. 51, pp. 2020 2040, Jun 2005.

[7] Y. Qi, R. Hoshyar, M. A. Muhamamd, and R. Tafazolli, "H²-ARQRelaying: spectrum and energy efficiency perspectives," IEEE JSAC, vol. 29, no. 8, pp. 1547-1558, Sep. 2011.

[8] P. Hu, C. W. Sung, and K. W. Shum, "Joint Channel-Network Coding for the Gaussian Two-Way Two-Relay Networks," EURASIP Journal on Wireless Communications and Networking, vol. 2010, pp. 1-13, Mar. 2010.

[9] L. J. Baik and S. Y. Chung, "Network Coding for Two-way Relay Channels Using Lattices," In Proc. of ICC'08, pp. 3898-3902, May 2008.

[10] B. Rankov, and A. Wittneben, "Achievable rate regions for the two-way relay channel," IEEE International Symposium on Information Theory, Jul. 2006, pp. 1668-1672.

[11] Z. Liu, S. Cheng, A. Liveris, and Z. Xiong, "Slepian-Wolf coded nested lattice quantization for Wyner-Ziv coding: high-rate performance analysis and code design," IEEE Trans. Inform. Theory, vol. 52, no. 10, pp. $4358-4379$, Oct. 2006.

[12] Ravi Narasimhan, "Individual outage rate regions for fading multiple access channels," in Proc of ISIT, pp. 1571-,1575, Jun. 2007.

[13] A. Sanderovich, S. Shamai, Y. Steinberg, and G. Kramer, "Communication Via Decentralized Processing," IEEE Trans. Inform. Theory, vol. 54, no. 7, pp. 3008-3023, Jul. 2008. 